\title{
THE CHAIN-STORE PARADOX REVISITED
}

\begin{abstract}
The rationality of predatory practices has been extensively debated among economists and lawyers. Selten (1978) considered a fictitious chain-store confronted with potential entrances of local competitors. In his formal analysis via an extensive game with complete and perfect information predatory behavior is precluded by the unique sequential (and perfect) equilibrium. Kreps and Wilson (1982b) and Milgrom and Roberts (1982) established in modified models with incomplete information that predation against early entrants may be rational since it creates a reputation to the effect that later potential entrants are deterred. The present paper offers a modification of Selten's model with complete but imperfect information which renders possible reputation and deterrence.
\end{abstract}

The rationality of predatory practices has been extensively debated among economists and lawyers. Selten (1978) considered a fictitious chain-store confronted with potential entrances of local competitors. In his formal analysis via an extensive game with complete and perfect information predatory behavior is precluded by the unique sequential (and perfect) equilibrium. Kreps and Wilson (1982b) and Milgrom and Roberts (1982) established in modified models with incomplete information that predation against eraly entrants may be rational since it creates a reputation to the effect that later potential entrants are deterred. The present paper offers a model with complete but imperfect information which renders possible reputation and deterrence.

\section{INTRODUCTION}

The conception of "predatory pricing" had a long tradition in economics and can in fact be traced back to Adam Smith (1776). Also jurisprudence has been concerned with predatory practices for quite a long time as the Sherman Anti-Trust Act from 1890 shows distinctly. There is no agreement in the literature on whether predatory pricing can be maintained as a viable threat and whether the anti-trust laws prohibiting predatory prices are necessary. The crucial point in this debate is the credibility of 
threats. It is therefore important to analyze in a purely game theoretic context whether effective threatening of potential entrants by a monopolist can be rational. Obviously, this is an abstract problem which may very well be treated in a model disregarding many aspects of real markets and details of actual pricing policies. Such a model was presented by Selten (1978). The plausibility of effective deterrence asserted by Selten is not confirmed by the formal game-theoretic analysis of his scenario. This is what Selten calls the "Chain-Store Paradox". Later work by Rosenthal (1981), Kreps and Wilson (1982b), and Milgrom and Roberts (1982) falls back upon and modifies Selten's model. The same I shall do in the present paper. Before decribing Selten's formal model in the next section I shall recall Selten's fictitious scenario of a market situation.

There are 20 towns, in each of which a chain-store, player $M$ for short, has exactly one branch. In each of these towns there is one potential competitor, player $k \in\{1, \ldots, 20\}$. It is assumed that at 20 consecutive points of time each of the 20 players $k \in\{1, \ldots, 20\}$ has to decide whether he prefers to use his capital in a different way. As soon as $k$ has decided, the other 20 players, $M, k^{\prime} \in\{1, \ldots, 20\}, k^{\prime} \neq k$, are informed about his choice. If $k$ has decided not to establish a shop, i.e. not to enter the $k$ th market, nothing changes on this market. In this case a reaction or decision of player $M$ is not necessary and, therefore, does not take place. If $k$ has decided to enter the $k$ th market then $M$ has to choose between two alternatives. Without going into details of the structure of possible pricing policies, it is assumed for the sake of simplicity that these two alternatives are described sufficiently well by the adjectives "cooperative" and "aggressive". Here "cooperative" describes peaceful reaction rather than proper cooperation. Also $M$ 's decision is immediately made known to all other players. Hence, whenever one of the players is to decide he knows the complete history. After $k$ 's decision not to enter the market or after $M$ 's decision in the $k$ th town, player $k+1$ has to make his choice $(k \in\{1, \ldots, 19\})$. It is assumed that all players are profit maximizers. For player $M$ this means that he wants to maximize the sum of the profits on all markets. Also all players are assumed to be rational in the sense that they are willing and able to conclude and compute correctly. As to the players' outcomes it is assumed that for $M$ the situation on the $k$ th market is optimal if he is a monopolist, i.e. if player $k$ decides not to enter the market. In case 
of entrance of player $k$ peaceful reaction of $M$ yields him a higher profit on this particular market than an aggressive policy. For player $k \in\{1, \ldots, 20\}$ it is assumed that entrance followed by peaceful reaction of $M$ is optimal. But renunciation of entrance is more profitable for him than entrance followed by a price war. Finally it is assumed that cooperation (in the proper sense) is precluded.

There is a straightforward way to model this scenario as a 20 -fold repetition of a two-person game in extensive form with complete and perfect information. Also the resulting repeated game is an extensive game with complete and perfect information.

Motivating my own modelling of the Chain-Store scenario requires knowledge of Selten's original model as well as of the modifications due to Kreps and Wilson and to Milgrom and Roberts. Accordingly, I shall briefly present and discuss these models in the next two sections. The indispensable technical tools are collected in the appendix.

Selten (1978) used for his analysis of the Chain-Store paradox the concept of a perfect equilibrium, which he introduced in (1975). Let me conclude this section by giving some reasons why I prefer the concept of sequential equilibrium, and why I do so in particular for the present analysis.

Not only are "most" sequential equilibria perfect, but sequential equilibria have also much nicer mathematical properties and are easier traceable (cf. Kreps and Wilson, 1982a). Perfect equilibria are sequential equilibria enjoying an additional weak robustness property. For stability considerations, however, which are completely out of the scope of the present paper, a much stronger robustness with respect to all distortions rather than only with respect to one would be desirable. According to Kohlberg and Mertens (1982) "Perfectness seems to be essentially equivalent to sequentiality plus efficiency". But the bad behavior of sequential equilibria with respect to dominance as compared with perfect equilibria has no impact on the rationality conception reflected by the equilibrium notion. Perfect equilibria do not represent aspects of rationality beyond the "sequential rationality" embodied in the concept of sequential equilibrium. Since understanding the Chain-Store paradox depends only on the rationality aspects of the prevailing equilibrium concept the use of sequential equilibrium seems to be adequate. 


\section{SELTEN'S CHAIN-STORE GAME}

The scenario described in the previous section is modelled by Selten as a 20-staged game tree composed of 20 identical two-person games played consecutively on the 20 markets. The $k$ th stage game is described by Figure 2.1.

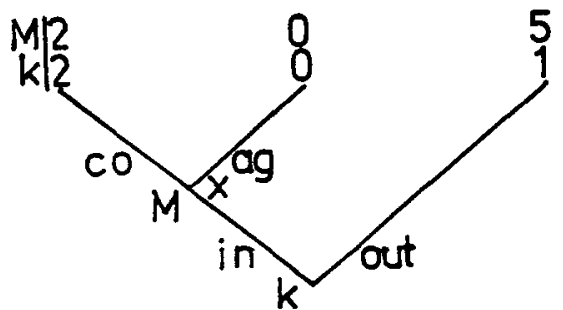

Fig. 2.1.

The decisions "enter", "stay out", "cooperative" and "aggressive" are abbreviated by "in", "out", "co", and "ag”, respectively. Although the specific choice of numbers is consistent with customary conceptions as to the relative sizes of monopoly and duopoly profits, for each player only the ordinal ranking of the outcomes independent of the opponents' outcomes are important.

If $M$ is to make his choice in this game he knows that $k$ chose to enter the market. As a rational profit maximizer $M$ therefore will play "co". Presuming this behaviour of $M$ it is rational for $k$ to enter the market. Indeed, the resulting equilibrium is sequential, whereas the other equilibrium, (out, ag) is not. That is, at each node of the game tree (in, co) induces an equilibrium on the partial tree originating from this specific node, while this is not the case for (out, ag) at node $x$. A formal definition of sequential equilibria is given in the appendix. Selten's analysis of the repeated game, the Chain-Store game, results in the assertion that the situation is the same as in the one-stage game. The unique sequential equilibrium prescribes entrance for all $k \in\{1, \ldots, 20\}$ and peaceful reaction for $M$. The induction argument is straight. Establishing the sequential equilibrium corresponds to finding an optimum in dynamic programming. The plausible "deterrence solution" is not supported by the analysis. This is what Selten calls the "Chain-Store Paradox".

The specific structure of the extensive games where no lacks of information are present, excludes the generation of reputation and keeps the 
actions on the 20 markets independent. In case of full information and rationality there is nothing to be learned. Player $M$ cannot make any potential entrant believe that he is a predator, since they know he is not. The common knowledge (cf. Auman, 1976; Milgrom, 1981) that cooperation is the unique sequentially rational reaction to entry and that entry is the unique sequentially rational choice, enforces the unique sequential equilibrium. This fact led Kreps and Wilson (1982b) and Milgrom and Roberts (1982) to modifications of Selten's model in which lacks of information are present.

\section{MODIFICATIONS WITH INCOMPLETE INFORMATION}

In this section, modifications of the Chain-Store Game due to Kreps and Wilson (1982b) and Milgrom and Roberts (1982) are discussed. I shall present simplified versions of their models which suffice to illustrate the basic idea of the modifications. I also adapt the payoffs to Selten's original setup.

Starting point for both these papers is the insight that relaxing of the complete information assumption destroys the presence of common knowledge and, therefore, leads to a breakdown of the backward induction employed by Selten. Only in the presence of lacks of information learning from experience and, thus, building up of reputation is possible.

Accordingly, Kreps and Wilson as well as Milgrom and Roberts altered Seltens scenario by assuming some doubt on side of the entrants about whether their perceived model of the game to be played is correct. Hence the entrants can consider the possibility that with a small probability they are not correctly informed about the true tree of the one-stage game. Either different outcomes for $M$ (Kreps and Wilson, 1982b) or different alternatives for $M$ (Milgrom and Roberts, 1982) are considered possible. In either approach there is agreement among the entrants on what might be the alternative game and on the probability of this alternative game being the true one. Also the way how to change subjective probabilities as a consequence of certain observations is in both models for all entrants the same. It turns out that in both models there are sequential equilibria prescribing predation in the first stages for $M$ and accordingly staying out of the market for nearly all potential entrants. The fear of a predatory response of $M$ keeps the potential entrants at almost all stages out of the 
markets. Only close to the end, where peaceful coexistence is more profitable than aggression the potential entrants cannot be deterred anymore. The fear of most entrants is justified in both models since there exists with positive probability a "strong" monopolist (cf. Figure 3.1.), who earns from predation even in the short run, or an "aggressive" monopolist (cf. Figure 3.2.), whose only possible reaction is predation.

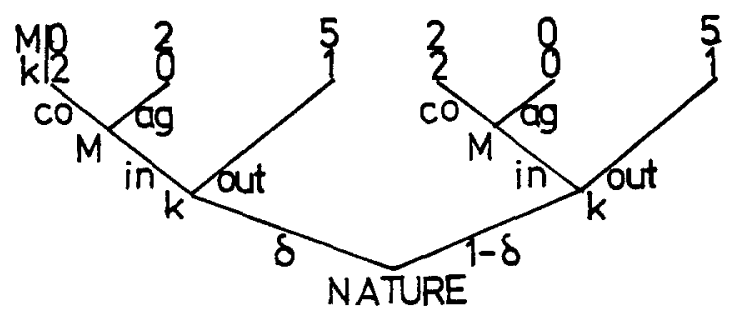

Fig. 3.1,

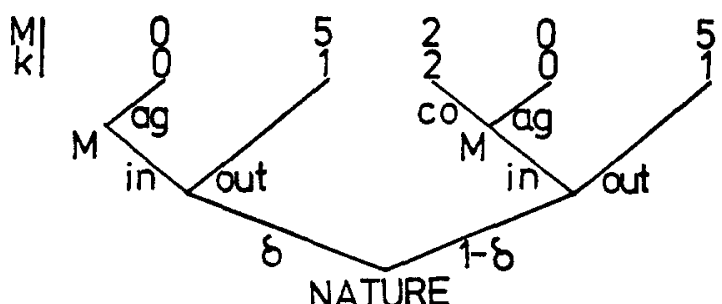

Fig. 3.2.

In contrast to Selten's model, where all information sets are singeltons the beliefs play an important role here. The potential entrants' common subjective probability that $M$ is a predator increases with the number of stages of the game in which $M$ responses aggressively. When this probability becomes large enough for player $k$ he will decide to stay out. If $k$ is small enough, i.e. if reputation grows fast enough, then aggression increases indeed $M$ s long run profit. Visualizing this fact prevents already the first players from entering the markets. Infact there are relations between the specification of initial beliefs, the number of stages of the game, the exact payoffs for the players, and the rule how beliefs are influenced by experience. By distinguishing "plausible" from "implausible" beliefs Kreps and Wilson as well as Milgrom and Roberts arrive at 
certain uniqueness results. Since this is not the point of the present paper I shall not discuss it. For me it is important, to what extent results do explain the paradox. From both models one can learn that a small lack of information about the game to be played suffices to avoid the appearance of the Chain-Store Paradox. Selten's model is the perhaps unrealistic limit case of the models just discussed, where all entrants' error probability degenerates to zero. Indeed, in many (when not in most) situations the incomplete information hypothesis seems to be much more realistic. Therefore, I think that nobody who wants to model problems of market entry should be harassed by the Chain-Store Paradox. Yet, I am not satisfied with the solution of the game theoretic paradox via incomplete information. If a complete information scenario as described in detail by Selten is taken for granted is than the Chain-Store paradox inevitable? Stated differently: Is the lack of information about what the opponent is going to choose, which is a fundamental characteristic of any game in which the prevailing solution concept fails to predict a unique outcome, sufficient to enable the construction of reputation via deterrence in a way compatible with the unbounded rationality of all players? I shall give an affirmative answer to these questions in the next section. This will be done by modifying Selten's model in such a way that the result will, as Wilson (1983) states it, "illustrate the general principle that reputations... can be used to coordinate selections of equilibria...".

\section{MODIFICATION WITH IMPERFECT INFORMATION}

Starting point for my consideration is the observation that the order in which players move in an extensive game may be arbitrary. A two-person game in normal form, where each player has two strategies, for instance, can be modelled in two different ways as an extensive game depending on who is the first to move. Accordingly the structure of information sets for the two players is different as are the possibilities of expressing beliefs formally. Obviously this may have consequences for the analysis in terms of sequential equilibria.

Looking at the one-stage game of Figure 2.1 again it becomes obvious that altering the order of moving would create an information set for player $k$ which consists of two nodes. This is illustrated in Figure 4.1. In this way the concept of beliefs, an important ingredient of sequential 


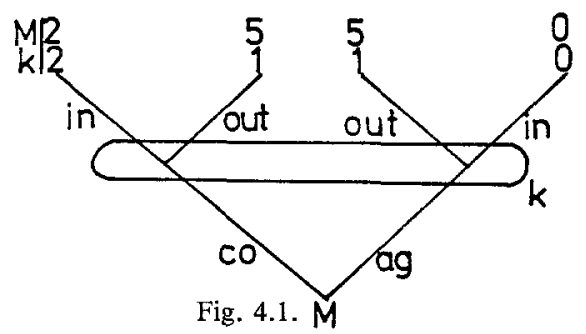

equilibrium, becomes available in the new game tree. I claim, that the new game is alse a correct model of the decisional problem in Selten's scenario. Clearly, it is not completely correct in every respect. In the original scenario player $M$ decides only if and after player $k$ has already entered the market. Therefore we have infact reversed time within every one-stage game. But time should play a role in the analysis only as far as information is concerned. Here it becomes important to follow von Neumann and Morgenstern (1944) who make a difference between anteriority and preliminarity of moves. In games with perfect recall (cf. Kuhn, 1953) preliminarity, i.e. an "informational before", implies anteriority, i.e. a "before in time", but not vice versa. The original set theoretic formalization of extensive games in chapter II of von Neumann and Morgenstern allows distinction of these properties. The more special formalization of extensive games by trees creates an asymmetry which sometimes may be impeding.

Let us look at the game in Figure 4.1. For player $k$ there is no change of information involved. He has to decide in ignorance of $M$ 's decision. Hence the decisional problem of $k$ is formalized with a new model as adequately as in the original one.

What about player $M$ ? Unless the very fact of deciding becomes an ingredient of the theory (for instance, deciding might be costly), $M$ can as well decide before $k$, since only in case of $k$ 's entrance his decision becomes effective. Any potential effect on players $k^{\prime}>k$ will be the same as in the original model.

The equivalence of the two models, the present one and Selten's original one, is based on the Sure Thing Principle (cf. Savage, 1954). In the new model there are two possible states of the world, "in" and "out", and $M$ has to choose between "co" and "ag" without knowing the true state. Whatever his preferences between "co" and "ag" in case of "in" 
is, say (co R ag), he is indifferent between "co" and "ag" in case of "out", because there is no difference between "co" and "ag" in the consequences. But then the Sure Thing Principle prescribes (co $\mathrm{R} \mathrm{ag}$ ) unconditionally. The irrelevance of state "out" for $M$ 's decision problem allows $M$ to make his choice as well before $k$ 's decision, anticipating his entrance.

But the employment of the Sure Thing Principle is totally legitimate and not new in our context. First of all is the Sure Thing Principle hidden in the Neumann-Morgenstern axioms for utility as Malinvaud (1952) has pointed out. Moreover, the acceptance of behavior strategies as equivalent to mixed strategies in games with perfect recall in Selten (1978), Kreps and Wilson (1982b), and Milgrom and Roberts (1982) reflects the idea that agents decide at each information set independently of their potential decisions at different information sets. Again the Sure Thing Principle is employed.

Let us look now for the sequential equilibria in the alternative one-stage game. Obviously (in, co) is again a sequential (even perfect) equilibrium with belief $\mu_{k}(c o)=1, \mu_{k}(a g)=0$ for player $k$. But now (out, ag) is a sequential equilibrium, too, since $\mu_{k}(c o)=0, \mu_{k}(a g)=1$ defines a consistent belief, based on which "out" maximizes the expected outcome for player $k$.

Certainly, the new situation in which (out, ag) is also a sequential equilibrium does not make it more plausible as long as the isolated one-stage game is considered. The situation changes, however, if one considers the 20 -fold repeated game.

Suppose players $1, \ldots, 7$ trust in the infallible logic telling them that the rational player $M$ must response peacefully. Assume furthermore that $M$ reacts seven times aggressively to entrance, although this seems to be irrational. What is then a reasonable choice for player 8 ? Shall also he trust this abstract argument and take the risk to be punished in the same way as his predecessors? Or shall he react to his past observations and stay out? Assume he chooses to stay out. Then he will be tempted to check whether this is consistent with his conviction that $M$ is rational. Now, if the deterring effect keeping player 8 out of the market lasts until player 16 has to decide, then the predatory strategy has yielded $M$ the total outcome of $9 \cdot 5=45$. But this exceeds the total profit of permant peacefull reaction to entrance, which is only $20 \cdot 2=40$ Hence player 8 's decision 
to stay out is rational, as is $M$ 's behaviour. Yet, in equilibrium it is already for players 1 to 7 better to stay out. In the new model a doubt whether $M$ will react peacefully does not imply automatically a doubt that $M$ is rational.

Clearly, the more stages the repeated game has the more time is available for building up reputation. Hence plausibility grows with the number of stages. A formal proof that such an equilibrium is indeed a sequential one is straightforward.

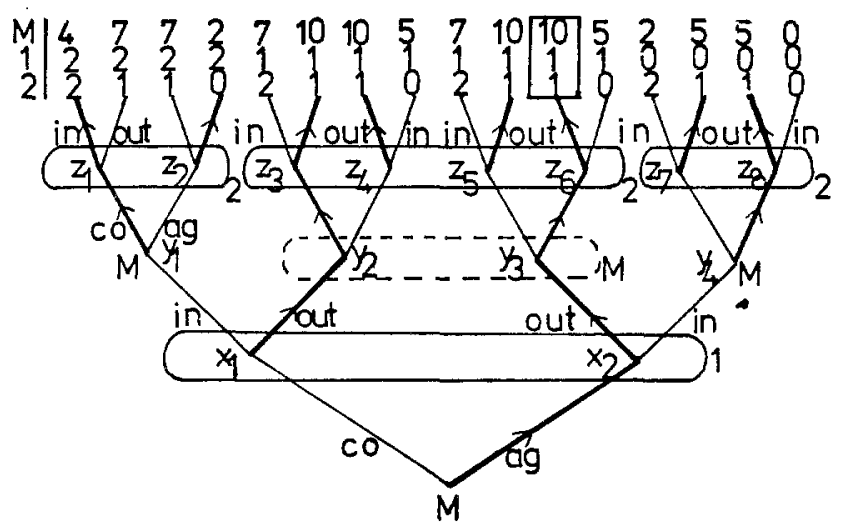

Fig. 4.2.

In Figure 4.2. a two-staged version of the modified Chain-Store game is pictured. It is not surprising that due to the few stages the illustrated sequential equilibrium which prescribes predation is not very plausible. The strategies and beliefs for the players in this example are as follows:

$M \quad$ play "ag" in the first stage; make in the second stage the same choice as in the first stage.

1 stay out; $\mu_{1}\left(x_{1}\right)=0, \mu_{1}\left(x_{2}\right)=1$

2 stay out, when $M$ played "ag" in the first stage, enter otherwise; $\mu_{2}\left(z_{i}\right)=0, i \in\{2,3,4,5,7\}, \mu_{2}\left(z_{i}\right)=1, i \in\{1,6,8\}$

Let us consider the game tree of Figure 4.2. If Player 1 stays out Player 2 does not know whether $M$ decides to response peacefully or aggressively to entrance on the first stage. Hence Player 2's information set consists of 4 nodes in this case, namely $z_{3}, z_{4}, z_{5}, z_{6}$. In this model player $M$ knows, however, at the second stage, what he decided in the first stage, even if 
Player 1 did not enter. As a consequence the unique point corresponding to "out" in the original game is split up now into two points which can be distinguished by $M$. If we build a new information set for $M$ consisting of the points $y_{2}, y_{3}$, as illustrated by the dotted line in Figure 4.2., then $M$ is in the same informational situation at Stage 2 as he was in the original game. However, then the new game is not anymore a game with perfect recall, since $M$ forgot his choice at the first stage before his move at the second stage. Although both versions allow for building up reputation, there are different sets of events with respect to which conditioning is possible. In Selten's model the monopolist cannot make decisions at a certain stage which are dependent on his own decision at a specific earlier stage unless there was entrance at this stage. Only in the case of entrance there was a decision which can be remembered at later stages and therefore be taken into consideration. In our present model $M$ has plans for every earlier stage in his drawer even if these plans have never been used. There is a fundamental difference in how past decisions can influence present or future decisions. Certainly, also in Selten's model $M$ can make a plan in which the decision at later stages depend on former decisions. The difference between the models as far as consideration of past decisions is concerned is the following. In Selten's model $M$ 's decision at Stage 7 may depend on his own decision at Stage 6. If this latter one is a random choice then the move at Stage 7 can only depend on the probability distribution prescribed at Stage 6 by $M$ 's behaviour strategy. It cannot depend, however, on the specific outcome of the random experiment performed at Stage 6, which is prescribed by this probability distribution. Yet, this dependence on the specific outcome is possible in the present alternative model. Here $M$ can make his decisions at all stages dependent on what really happened in the past, i.e. there are much more potential conditioning events for later decisions.

\section{CONCLUSIONS}

The Chain-Store Paradox led Selten (1978) to the conclusion that the unbounded rationality approach of game theory which failed to support the plausible outcome, should be abandoned in favor of a bounded rationality approach. 
Kreps and Wilson (1982b) and Milgrom and Roberts (1982) showed that a slight modification of Selten's scenario can be modelled in such a way that the Chain-Store Paradox is avoided.

In the present paper it is shown that it is not even necessary to change the original scenario to avoid the paradox. One has only to choose a suitable one among some equivalent models.

Let me note in passing that also the one-stage game in Figure 5.1. is equivalent to Selten's original one.

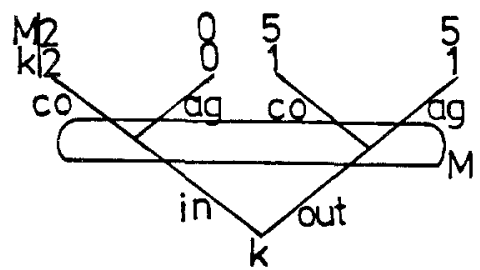

Fig. 5.1.

It is equally suitable for avoiding the paradox. However, intuitively the model presented in Section 4 seems more appealing since it is more adequate to have the entrants formally endowed with beliefs rather than the monopolist.

I intentionally did not present an explicit learning model which realizes reputation building and deterrence. It could easily be established similar to those in Kreps and Wilson (1982b) and Milgrom and Roberts (1982). Like these it would be quite plausible but still adhoc. My point that reputation building and deterrence is possible also in Selten's original scenario by alternative modelling is, so I hope, clear enough.

Let me compare my alternative model with the incomplete information models sketched in Section 3. An obvious drawback in those models is that, as Kreps and Wilson (1982b, p. 276) write, "by clearly choosing the nature of that small uncertainty (pricesely - its support), one can get out of a game-theoretical analysis whatever one whishes." A good example for this claim is provided by Kreps et al. (1982), a proof for a corresponding theorem is given by Maskin and Fudenberg (1983). ${ }^{1}$ The situation is, however, totally different in my complete information model. Here reputation can help only to select from different sequential equilibria. The only uncertainty concerns which of several possible equilibrium strategies other players chose. There is no uncertainty about the game which is to 
be played. One can take the basic data of the game for granted and still get reputation. And here we are in contradiction to the prevailing view that common knowledge precludes building up of reputation. Milgrom and Roberts (1982) claim that "informational asymmetries... will be necessary for reputation building to occur in general..." And Dixit (1982) writes: "For reputation to have a role, therefore, we need either infinite (or indefinite) repetition, or incomplete information."

There is certainly common knowledge about the game in my model and hence there are no informational asymmetries. Also there is complete information. Yet, reputation has a role there. However, if one extends the common knowledge or informational asymmetry requirements to what players know about other player's choices then common knowledge requires uniqueness of sequential equilibrium in a game with complete and perfect information. Selten's original game is an example. Clearly, reputation building is impossible in such a case.

I would like to emphasize at this point that the equilibrium (in, co) remains the unique perfect equilibrium also in my model. The rationality properties of perfect equilibria are compatible with building reputation in Selten's scenario. The robustness property is not. This confirms a point made by Kreps and Wildon (1982a) who state: "The power of reputation seems to be positively related to its fragility."

The previous paragraph is not to be interpreted as if perfect equilibria exclude reputation building. Also here non-uniqueness is the key. Whatever equilibrium or solution concept is used non-uniqueness opens the door for reputation.

In Selten's original model there is a unique sequential equilibrium. The term "Chain-Store Paradox" expresses the fact that in this model there is an overdetermination via the employed concept of sequential equilibrium since plausible outcomes are ruled out. In my alternative model there is abundance of sequential equilibria which may be critizised as an underdetermination, since many implausible outcomes are not ruled out. The same holds true for the incomplete information approach. Kreps and Wilson (1982b) as well as Milgrom and Roberts (1982) seem to consider restriction to certain "plausible" equilibria a remedy. In fact by these means they get uniqueness on the equilibrium path. My point of view is somewhat different. I consider the multiplicity of equilibria an advantage rather than a drawback. It restricts the set of rational outcomes without 
determining this set as a singleton. Several people may differ not only in tastes but also in other characteristics which are involved in learning processes. Not all people are impressed in the same way by the same event. Credulity, optimism, scepticism, obstinacy may influence the building of beliefs dependent on events. This may lead to very different equilibrium outcomes all of which are based on rational behaviour of the players. Somebody's belief that doomsday is tomorrow may be considered implausible by others but if he behaves accordingly he may do in a completely rational way. I think uniqueness of social equilibria is a fiction. I interpret equilibrium concepts as advices precluding nonrational non-equilibrium behavior rather than as concrete devices how to choose. Further restriction of the set of sequential equilibria should not be accomplished in my opinion by ad hoc postulates. Rather I would like further restriction via a theory which explicitly explains learning processes. I feel that the concept of sequential equilibrium provides an appropriate game theoretic a priori restriction to rational outcomes.

It would be interesting to see what kind of outcomes would appear if the Chain-Store game is played in experiments. In particular it would be informative to see whether results differ according to whether players are confronted with Selten's game or with my modification. If they would differ then such game experiments would cast additional doubts on the justification of independence axioms representing the Sure Thing principle.

A further very interesting question arising from the above considerations is, how information patterns influence sequential equilibrium analysis. Equivalence of information patterns has been analyzed by Dalkey (1953). It would be interesting to have a notion of equivalence of games under sequential equilibrium analysis. One can criticize the prevailing equilibrium concepts because of their sensitivity with respect to (seemingly) inessential modifications of the game tree (cf. Kohlberg and Mertens, 1982). One can, however, criticize the modelling of extensive games. It would be desirable to have a canonical way of representing an extensive game in which simultaneous moves are possible and where preliminarity is modelled in a natural way not based on anteriority. This might require to go back to von Neumann's and Morgenstern's set theoretic modelling of extensive games. 


\section{APPENDIX}

I assume that the notion of extensive game and its representation as a tree are known. For definitions see Kuhn (1952), Luce and Raiffa (1976), and Selten (1975). Throughout the paper only games with perfect recall are considered, i.e. games in which every player does not forget anything he knew at former stages of the game.

A game $\Gamma$ is a game with perfect recall if the following holds true: Consider any two nodes $x, y$ of the game tree being in the same information set of a certain player. If two paths start at $x$ and $y$ by different moves, then any subsequent information set of that player can be met by at most one of these two paths.

A pure strategy of a player is a specification of choice at each of the players information sets. A mixed strategy of a player is a probability distribution on the set of his pure strategies. A behavior strategy for a player is an assignment of a probability distribution over the possible choices at each of the players information sets. Kuhn (1953) has shown that any outcome which is possible via mixed strategies in a game with perfect recall can as well be realized via behavior strategies.

A (Nash-)equilibrium of an $n$-person game $\Gamma$ with perfect recall is a combination of behavior strategies of all players, each of which is a best response to the whole of the others. "Best" means yielding the highest expected payoff.

A system of beliefs assigns to each of every player's information sets a probability distribution on this set. An assessment is a pair $(b, \mu)$, where $\mu$ is a system of beliefs and $b$ a combination of behavior strategies for the $n$ players. If $b$ has the property that all nodes in all information sets have positive probability, i.e. if $b$ is completely mixed, then it defines on each information set a conditional probability given this information set. In this way $b$ generates a specific system of beliefs, $\mu(b)$. An assessment $(\bar{b}, \bar{\mu})$ is consistent if there is a sequence of completely mixed combinations of behaviour strategies, $b_{n}$, converging (pointwise) to $\bar{b}$, such that $\mu\left(b_{n}\right)$ converges (pointwise) to $\bar{\mu}$. A combination of behavior strategies, $b$, is a sequential best response to the assessment $(\bar{b}, \mu)$ if $b$ defines for every player at any of his information sets a best response to $(\bar{b}, \mu)$. Again "best" means maximizing the expected payoff with respect to $\bar{b}$ and $\mu$. 
A consistent assessment $(b, \mu)$ for which $b$ is a sequential best response to $(b, \mu)$ is a sequential equilibrium. A combination of behavior strategies $b$ is also called sequential equilibrium, if there exists a system of beliefs, $\mu$, such that $(b, \mu)$ is a sequential equilibrium.

A sequential equilibrium $b$ is a perfect equilibrium if there is a sequence of completely mixed combinations of behavior strategies, $b_{n}$, converging to $b$ such that $b$ is a sequential best response to $\left(b_{n}, \mu\left(b_{n}\right)\right)$ for all $n$.

In a sequential equilibrium no player has a reason to regret his choice whenever he has to move, even off the equilibrium path. A sequential equilibrium prescribes optimal choices even at points never arrived at if the prescription is followed by all players. Tracing a sequential equilibrium amounts to applying Bellman's optimality principle and can be done by dynamic programming. The beliefs are consistent via Bayes rule with eachother and with the equilibrium combination of behavior strategies whenever Bayes rule can be applied.

A perfect equilibrium is a sequential equilibrium, which even if all players modify slightly their equilibrium strategies in a suitable specified way, for every player remains a best response to the modified strategies of the others.

\section{ACKNOWLEDGEMENT}

Without implicating them I gratefully acknowledge helpful comments of a number of people including especially Beth Allen, Robert Aumann, Eric van Damme, Pradeep Dubey, Martin Hellwig, Reinhard John, Ehud Kalai, Morton Kamien, Laurenz Kohlleppel, Robert Rosenthal and Reinhard Selten.

\section{NOTE}

1 A natural step then is to leave the game-theoretic context and to treat the problem "like one-player decision problem"' (Rosenthal, 1981, p. 92).

\section{REFERENCES}

Aumann, R. J.: 1976, 'Agreeing to Disagree', The Annals of Statistics 4, 1236-1239.

Aumann, R. J., and Maschler, M.: 1972, 'Some Thoughts on the Minimax Principle', Management Science 18, P54-P63. 
Dalkey, W.: 1953, 'Equivalence of Information Patterns and Essentially Determinate Games', in Kuhn H. W. and Tucker, A. W. (eds.), Contributions to the Theory of Games, Vol. 2, pp. 217-243, Princeton University Press, Princeton, N.J.

van Damme, E.E.C.: 1983, Refinements of the Nash Equilibrium Concept, Springer Verlag, Heidelberg.

Dixit, A.: 1982, 'Recent Developments in Oligopoly Theory', The American Economic Review 72, 12-17.

Kohlberg, E. and Mertens, J. F.: 1982, 'On the Strategic Stability of Equilibria', Econometrica, forthcoming.

Kreps, D. M., Milgrom, P., Roberts, J., and Wilson, R.: 1982, 'Rational Cooperation in the Finitely Repeated Prisoners Dilemma', Journal of Economic Theory 27, 245-252.

Kreps, D. M. and Wilson, R.: 1982a, 'Sequential Equilibria', Econometrica 50, 863-894.

Kreps, D. M. and Wilson, R.: 1982b, 'Reputation and Imperfect Information', Journal of Economic Theory 27, 253-279.

Kuhn, H. W.: 1953, 'Extensive Games and the Problem of Information', in Kuhn H. W. and Tucker, A. W. (eds.), Contributions to the Theory of Games, Vol. 2, pp. 193-216, Princeton University Press, Princeton, N.J.

Luce, R. D., and Raiffa, H.: Games and Decisions, Wiley, New York.

Malinvaud, E.: 1952, 'A Note on von Neumann-Morgenstern's Strong Independence Axiom', Econometrica 20, 679.

Maskin, E, and Fudenberg, D.: 1983, 'The Perfect Folk Theorem for Finitely-Repeated Two-Person Games with Incomplete Information', Econometrica, forthcoming.

Milgrom, P.: 1981, 'An Axiomatic Characterization of Common Knowledge', Econometrica 49, 219-222.

Milgrom, P, and Roberts, J.: 1982, 'Predation, Reputation and Entry Deterrence', Journal of Economic Theory 27, 280-312.

Rosenthal, R. W.: 1981, 'Games of Perfect Information, Predatory Pricing and the Chain-Store Paradox', Journal of Economic Theory 25, 92-100.

Savage, L. J.: 1954, The Foundations of Statistics, Wiley, New York.

Selten, R.: 1975, 'Reexamination of the Perfectness Concept of Equilibrium in Extensive Games', International Journal of Game Theory 4, 25-55.

Selten, R.: 1978, 'The Chain-Store Paradox', Theory and Decision 9, 127-159.

Smith, A.: 1776, An Enquiry into the Nature and Causes of the Wealth of Notions, Methnon and Co, London.

von Neumann, J., and Morgenstern, G.: 1944, Theory of Games and Economic Behaviour, Princeton University Press, Princeton.

Wilson, R.: 1983, Reputation in Games and Markets, Institute for Mathematical Studies in the Social Sciences, Technical Report No. 434, Stanford.

Universität Bielefeld,

Institute of Mathematical Economics,

Postfach 8640,

Bielefeld 1,

F.R.G. 